\section{Dynamic Threshold for Radiation Target Volume by PET/CT $^{5}$}

TO THE EDITOR: The findings of Biehl KJ et al. (1) are interesting but not unexpected. It has long been proposed, following our prior study on phantoms and patients with non-small cell lung cancer, that a dynamic threshold rather than a single constant threshold is required for target volume definition (2). The constantthreshold method could significantly underestimate the extent of target volume (2). Although Biehl et al. (1) found a similar trend toward an increasing threshold percentage value with a decreasing standardized uptake value (SUV) and gross tumor volume (GTV) measured by CT, they failed to address the potentially larger SD when the SUV approaches a low value, especially when the mean SUV is less than 2.0 (2). In their series, 4 patients had tumors smaller than $3.0 \mathrm{~cm}$ with a mean maximum SUV ( \pm SD) of $3.0 \pm 0.4$. The mean SUV of the tumor in some of these 4 patients would be expected to be below 2.0. In addition, partial-volume effects start to set in at a size of less than $2 \mathrm{~cm}$ for most of the current PET/CT cameras. This was especially true in view of the fact that the smallest tumor studied in their series was only $1.2 \mathrm{~cm}$. Thus, the data obtained empirically for these 4 patients should be interpreted cautiously.

On a detailed review of the entire series of patients in our prior study (2), the greater difference seen in tumors has been attributed to the greater heterogeneity in the SUV of tumors than in the SUV of phantom spheres. In other words, because many tumor volumes have inordinately high maximum-point intensities, despite stable mean intensities, the constant-threshold method drives the threshold upward and results in a smaller volume. Our conclusion is that the mean tumor SUV appears to be a more stable parameter than the maximum SUV, on which the constant method, using varying percentages of maximum intensity, is based. We wonder whether the study of Biehl et al. (1) has investigated the mean SUV as well, instead of just the maximum SUV. The inherent relationship between the metabolic tumor activity and the target volume threshold appears to be the mean SUV, which depends on both the metabolic activity and the physical size of the tumor (2). The mean SUV has been shown to be the most important parameter driving selection of a threshold SUV, and this threshold SUV can be defined dynamically according to the regressive functions determined by phantom calibration and patient data (2). In target/phantom delineation, deviation is less and accuracy greater when the proposed regressive threshold function is used than when a constantthresholding method is used (e.g., percentage of maximum intensity or constant SUV). After all, no true gold standard exists for GTV. Furthermore, current PET/CT is full of uncertainties about actual tumor sizes and metabolic values because of factors such as partialvolume effects, respiratory motion (3), imperfect CT attenuation mapping for PET, image reconstruction (4), and various other factors affecting SUV (5).

The study of Biehl et al. (1) emphasizes dependence on CT-GTV $\left(\mathrm{cm}^{3}\right)$ : threshold (percentage of maximum SUV) $=59 \times \log (\mathrm{CT}-$ GTV) -18 . In contrast, our prior study (2) emphasizes dependence on the functional parameter of metabolic rate: threshold (percentage of mean SUV) $=31+59 /($ mean SUV $)$. The former method assumes an accurate measurement of physical tumor size by CT, whereas the latter is based on an iterative model obtained by regression analysis using threshold SUV versus mean SUV (2). The threshold SUV does not assume an accurate measurement of tumor size but is dynamically determined from the unique point at which a nonlinear curve, representing various measured mean SUVs around a patient's tumor, intersects with a linear calibration line from the phantom data (2). Conceptually, this individualized dynamic metabolic threshold transcends the anatomic boundary, especially when the real GTV is unknown. Thus, the new (PET/CT) GTV should be a function containing the biochemical domain with some physical constraints, projecting into a real combined range of GTV bound by clinical tumor volume. For the time being, caution should be used, specifically in the case of tumor volumes with a mean SUV below or near 2.0, because of an uncertainty in target surface localization. If one uses the metabolic mean SUV, background activity and target volume do not independently affect selection of the appropriate threshold because the mean SUV calculation already includes the effects of both background activity and target volume. Therefore, the use of mean SUV in the regressive function from our prior study (2) already incorporates the varying-threshold method proposed by Biehl et al. (1) and other investigators (6), with consideration of both metabolic activity and physical size using PET/CT measurement. The dynamically derived mean SUV may be better applied as a unique and stable parameter to determine the threshold SUV for target volume delineation by PET/CT (2).

\section{REFERENCES}

1. Biehl KJ, Kong F-M, Dehdashti F, et al. ${ }^{18}$ F-FDG PET definition of gross tumor volume for radiotherapy of non-small cell lung cancer: is a single standardized uptake value threshold approach appropriate? J Nucl Med. 2006;47:1808-1812.

2. Black QC, Grills IS, Kestin LL, et al. Defining a radiotherapy target with positron emission tomography. Int J Radiat Oncol Biol Phys. 2004;60:1272-1282.

3. Erdi YE, Nehmeh SA, Pan T, et al. The CT motion quantitation of lung lesions and its impact on PET-measured SUVs. J Nucl Med. 2004;45:1287-1292.

4. Jaskowiak CJ, Bianco JA, Perlman SB, Fine JP. Influence of reconstruction iterations on ${ }^{18}$ F-FDG PET/CT standardized uptake values. J Nucl Med. 2005;46: 424-428.

5. Wong CY, Thie J, Gaskill M, et al. Addressing glucose sensitivity measured by F-18 FDG PET in lung cancers for radiation treatment planning and monitoring. Int J Radiat Oncol Biol Phys. 2006;65:132-137.

6. Nestle U, Kremp S, Schaefer-Schuler A, et al. Comparison of different methods for delineation of ${ }^{18} \mathrm{~F}$-FDG PET-positive tissue for target volume definition in radiotherapy of patients with non-small cell lung cancer. $J$ Nucl Med. 2005;46:1342-1348.

DOI: $10.2967 /$ jnumed.106.038141
Ching-Yee Oliver Wong
Paresh Mahajan

Di Yan

William Beaumont Hospital

Royal Oak, Michigan 